\title{
Prediction of post-resection prognosis with ADV score for huge hepatocellular carcinoma $\geq 13 \mathrm{~cm}$
}

\author{
Shin HWANG*, Ki-Hun KIM, Deok-Bog MOON, Chul-Soo AHN, Tae-Yong HA, Gi-Won SONG, Dong-Hwan JUNG, Gil-Chun PARK \\ Department of Surgery, Asan Medical Center, University of Ulsan College of Medicine, Seoul, Korea
}

Introduction: Multiplication of $\alpha$-fetoprotein, des- $\gamma$-carboxy prothrombin and tumor volume (ADV score) is a surrogate marker for post-resection prognosis of hepatocellular carcinoma (HCC). The objective of this study was to validate the predictive power of ADV score-based prognostic prediction model for patients with solitary huge HCC.

Methods: Of 3,018 patients, 100 patients who underwent hepatic resection for solitary HCC $\geq 13 \mathrm{~cm}$ during the study period between 2008 and 2012 were selected.

Results: Median tumor diameter and tumor volume were $15.0 \mathrm{~cm}$ and $886 \mathrm{~mL}$, respectively. Tumor recurrence and overall survival (OS) rates were $70.7 \%$ and $66.0 \%$ at 1 year, and $84.9 \%$ and $34.0 \%$ at 5 years, respectively. Microvascular invasion was the only independent risk factor for disease-free survival (DFS) and OS. DFS and OS stratified by ADV score with 1log intervals showed significant prognostic contrasts $(p=0.007$ and $p=0.017$, respectively). DFS and OS stratified by ADV score with a cutoff of 8log showed significant prognostic contrasts $(p=0.014$ and $p=0.042$, respectively). Combination of MVI and ADV score with a cutoff of 8 log also showed significant prognostic contrasts in DFS $(p<0.001)$ and OS $(p=0.001)$ according to the number of risk factors.

Conclusions: The prognostic prediction model with ADV score with or without combination of MVI could reliably predict the risk of tumor recurrence and long-term patient survival outcomes in patients with solitary huge HCCs $\geq 13 \mathrm{~cm}$. Results of this study suggest that our prognostic prediction models can be used to guide surgical treatment and post-resection follow-up for patients with huge HCCs. 